\title{
Uso de probióticos en alimentación animal
}

\author{
Use of probiotics in animal feed \\ Gutiérrez Castro Litsy ${ }^{1}$ y Güechá Castillo Andrea ${ }^{2}$ \\ ${ }^{1}$ Médico Veterinario Zootecnista, (c)MSc, Universidad de los Llanos y \\ ${ }^{2}$ Médico Veterinario Zootecnista, Universidad de los Llanos \\ litsy.gutierrez@unillanos.edu.co
}

Recibido 10 de Mayo 2016, Aceptado 29 de Octubre 2016

\section{RESUMEN}

Estudios realizados en los últimos años denotan que el uso de probióticos implementados en la dieta animal demuestra un óptimo aumento sobre los parámetros productivos en la producción de animales rumiantes y no rumiantes destinados a satisfacer la alta demanda que se presenta en proteína de origen animal. La implementación de los probióticos en la dieta para animales de producción se perfila como una opción positiva para el productor mejorando indicadores productivos y económicos, puesto que entra a competir con el uso exagerado de antibióticos, lo cual está afectando el rendimiento productivo animal y la utilidad empresarial. En el presente trabajo se recopila información sobre el uso de probióticos como alternativa para optimizar los parámetros zootécnicos con el fin de disminuir pérdidas económicas sin desmejorar el valor nutricional del producto final garantizando al consumidor la entrega de un producto inocuo y de buena calidad.

Palabras clave: Microorganismos benéficos, microbiota, dieta animal, alimento funcional.

\section{ABSTRACT}

Studies in recent years, show that the use of implemented probiotics in animal diet, demonstrate an optimal increase on performance in the production of ruminants and not ruminants intended to meet high demand occurs in protein of animal origin. The implementation of the probiotics in the diet for production animals is emerging as a positive choice for the producer improving economic and productive 
indicators, because it enters to compete with the overuse of antibiotics which is affecting the animal productive yield and business profit. In this paper, information is collected about the use of probiotics as an alternative to optimize the zootechnical parameters with the final purpose to reduce economic losses without deteriorating the nutritional value of the final product guaranteeing at the consumer the delivery of a harmless and good quality product.

Keywords: Beneficial microorganisms, microbiota, animal diet, functional food.

\section{RESUMO}

Estudos realizados em anos recentes denotam que 0 uso de probióticos implementados em dietas para animais demonstram um excelente aumento no desempenho em a produção dos animais ruminantes e não ruminantes projetados para atender a alta demanda que se apresenta em proteína de origem animal. A implementação de probióticos na dieta para animais de produção está emergindo como uma opção positiva para o produtor melhorando indicadores econômicas e produtivas, uma vez que entra a competir com o uso exagerado de antibióticos, que está afetando o rendimento produtivo animal e a rentabilidade das empresas. Neste trabalho é recolhido informação sobre o uso de probióticos como alternativa para optimizar os parâmetros zootécnicos a fim de reduzir as perdas económicas sem diminuir o valor nutricional do produto final garantindo ao consumidor a entrega de um produto seguro e de boa qualidade.

Palavras-chave: Microorganismos benéficos, microbiota, alimentação animal, alimento funcional.

\section{INTRODUCCIÓN}

En países tropicales como Colombia, las materias primas empleadas en la alimentación de animales de granja se caracterizan por presentar marcadas fluctuaciones en su cantidad y calidad, generando alteraciones en los ciclos productivos; además las dietas implementadas tradicionalmente con granos (soya), cereales (maíz) y aceites, no son aprovechados apropiadamente por el tracto digestivo, generando una menor efectividad nutricional y productiva en el 
animal, y en adición estos cultivos (cereales y granos) son considerados un mercado competitivo con respecto a la demanda en el país para suplir las necesidades de la alimentación humana (Eicher, 2005).

En la actualidad la industria pecuaria busca alternativas que permitan mejorar los parámetros zootécnicos con el fin de disminuir pérdidas económicas sin desmejorar el valor nutricional del producto final, garantizando el estado sanitario y buen rendimiento de los animales de carne que generen resultados económicos y rentables (Samaniego et al., 2007). Estos sistemas pecuarios se han especializado en generar una alta intensidad productiva, por lo que frecuentemente se han venido usando aditivos no nutricionales en la dieta, como es el caso de los antibióticos que son considerados promotores del crecimiento; sin embargo, su uso continuo ha favorecido el desarrollo de cepas patógenas resistentes y efectos residuales en los alimentos (carne, leche, huevo), que afectan no solo al consumidor, sino que además su utilización genera alteraciones y daños en la microbiota gastrointestinal del animal predisponiéndolo a enfermedades. Este panorama ha conllevado a la necesidad de buscar e investigar nuevas alternativas seguras e inocuas (García et al., 2005).

Estudios realizados en los últimos años, denotan que el uso de probióticos implementados en la dieta de los animales, demuestra un óptimo aumento sobre los parámetros productivos de rumiantes y no rumiantes que están destinados a satisfacer la alta demanda que se presenta por la proteína de origen animal (Bazay, 2010); por lo tanto, el uso eficiente de probióticos en la dieta alimenticia para animales de granja se perfila como una opción positiva para el productor no solamente desde el punto de vista biológico sino que también económico, debido a que entra a competir con el uso exagerado de antibióticos, que afecta la inocuidad de los productos pecuarios (Loddi et al., 2000).

\section{ALIMENTOS FUNCIONALES}

Los alimentos funcionales son aquellos compuestos que sean o no un nutrimento, tienen efectos positivos sobre uno o varias funciones del organismo propiciando 
bienestar en el animal. Los prebióticos y probióticos se consideran alimentos funcionales que son capaces de alterar la composición de la microflora gastrointestinal (Figueroa et al., 2005).

\section{Diferencia entre probióticos y prebióticos}

Los prebióticos: Son fructo-oligosacáridos, que sirven de nutrientes para la supervivencia y multiplicación de los probióticos, su función principal es estimular la acción bacteriana y son conocidos como estimulantes del crecimiento de bifidobacterias y lactobacilos, las cuales después de un corto periodo de ingestión del prebiótico, predominan en el intestino, ya que durante su desdoblamiento digestivo producen una serie de sustancias (ácidos grasos de cadena corta) que proveerán un medio ácido para los lactobacilos (Sarmiento, 2006).

Los probióticos: Son microorganismos vivos, es decir un cultivo viable de uno o varios microorganismos, que al ser administrados en dosis adecuadas en una dieta contribuyen a mejorar el balance y equilibrio microbiano, se activan una vez que colonizan el intestino, afectando benéficamente al hospedero porque optimiza las propiedades de la microbiota endógena; además también influyen sobre el sistema inmunológico del intestino y generan un aumento en: 1) La competencia por nutrimentos con otro tipo de flora intestinal; 2) La secreción de mucina protectora del intestino; y 3) La competencia por receptores de sitios de adhesión de la mucosa intestinal, inhibiendo el crecimiento de algunas especies de enteropatógenos (Ángel, 2014).

Los probióticos y prebióticos se comercializan en diferentes formas de presentaciones comerciales que pueden ser en forma de cultivos vivos como el yogur probiótico o en forma inactiva (pero vivos), como los que se encuentran en capsulas o comprimidos y por último en medio liquido fermentado. En la Figura 1, se observan algunos ejemplos de presentación de levadura en polvo como el Saccharomyces cerevisiae en el marguen izquierdo y en el marguen derecho Bacillus Subtilis en presentación líquida. 


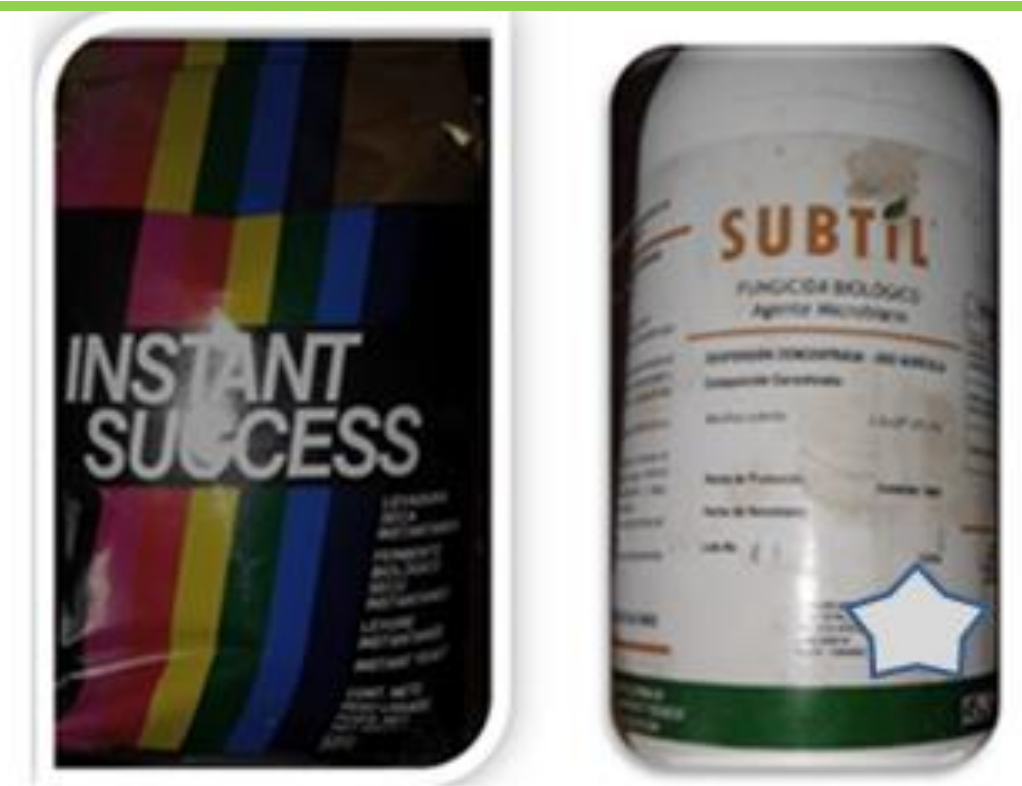

Figura 1. Presentación comercial de probióticos en el mercado. Fuente: Gutierrez y Güechá, (2016).

La mayoría de las bacterias que se utilizan como probióticos en los animales de interés zootécnico pertenecen a los géneros Lactobacillus sp, Enterococcus sp y Bacillus $s p$, aunque también se utilizan levaduras como Saccharomyces cerevisiae y hongos como Aspergillus oryzae (Angel, 2013). En la Figura 2 se ilustra un resumen de las principales características del uso de probióticos en las dietas, de las especies más utilizadas anteriormente mencionadas.

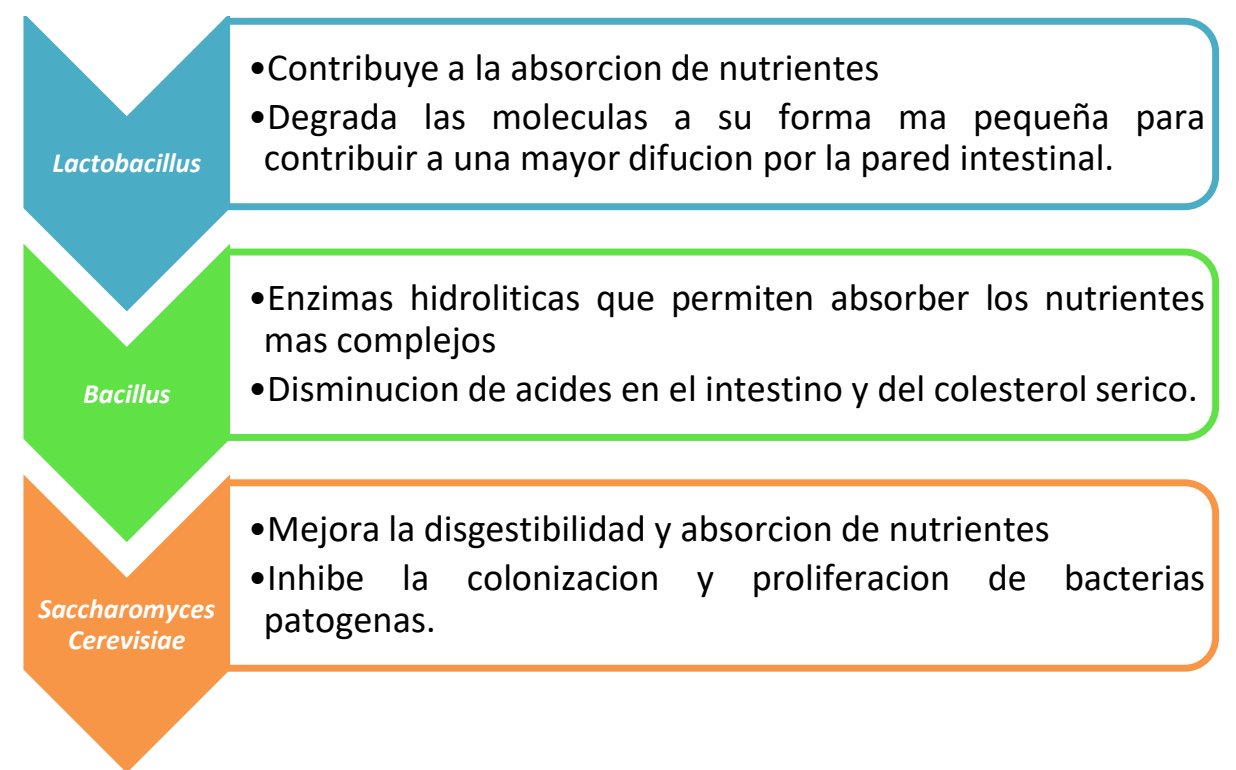

Figura 2. Características del uso de probióticos en las dietas. Fuente: Adaptado de Milian et al., (2008); Molina (2008); Miazzo (2005). 
Cuando se incorporan probióticos en la dieta para animales se pueden esperar resultados como: 1) Prevención de enfermedades que permite controlar la, bacterias (Salmonella sp, Escherichia coli), hongos y protozoos, contribuyendo a la seguridad sanitaria, 2) mejor conversión alimenticia, 3) disminución de costos de producción, 4) disminución de la mortalidad, 5) fortalecimiento del sistema inmunológico, y 6) se reduce ciclos fisiológicos y productivos, sin la aplicación de otra clase de aditivos no nutricionales como antibióticos (Blajmana et al., 2005)

\section{Comparación entre antibióticos y probióticos}

El rápido crecimiento de la población mundial ha generado un incremento en los sistemas de producción intensiva de animales como aves y cerdos que son de ciclos cortos de producción, por lo tanto, se ha aumentado su demanda para satisfacer las necesidades de proteína animal. En Colombia, el desarrollo de estas industrias se hace bajo las mejores condiciones de sanidad, sin embargo, el recién nacido no adquiere de manera rápida una población adecuada de microorganismos intestinales que permitan desarrollar su sistema inmunológico, siendo un blanco fácil contra diversas enfermedades gastrointestinales como lo son salmonelosis y coccidiosis (Lara y Burgos, 2012).

Por otro lado, el uso indiscriminado de antibióticos en los sistemas pecuarios por el concepto erróneo de los productores, quienes asumen que la administración temprana de estos aditivos evitará problemas de salud y corregirán las deficiencias de crecimiento, pero en realidad este tipo de actividades han traído consecuencias negativas debido a que estos medicamentos han perdido su eficiencia por la alta resistencia microbiana que se ha generado y los efectos residuales que se han presentado, convirtiéndose en un problema para el productor debido a la disminución en los índices zootécnicos y el aumento desmesurado en los costos de producción (Rigobelo et al., 2011).

En la Figura 3 se ilustra algunos efectos sobre el animal bajo del uso de probióticos en comparación con los antibióticos, donde se muestra que el uso de los primeros es una elección positiva actual sin efectos secundarios que permite 
optimizar la salud y a su vez mejorar los parámetros productivos, además de competir contra el uso indiscriminado de antibióticos que son empleados como promotores de crecimiento, por lo tanto estos alimentos funcionales (probióticos) se han vuelto una alternativa segura para los animales, los consumidores y el medio ambiente.

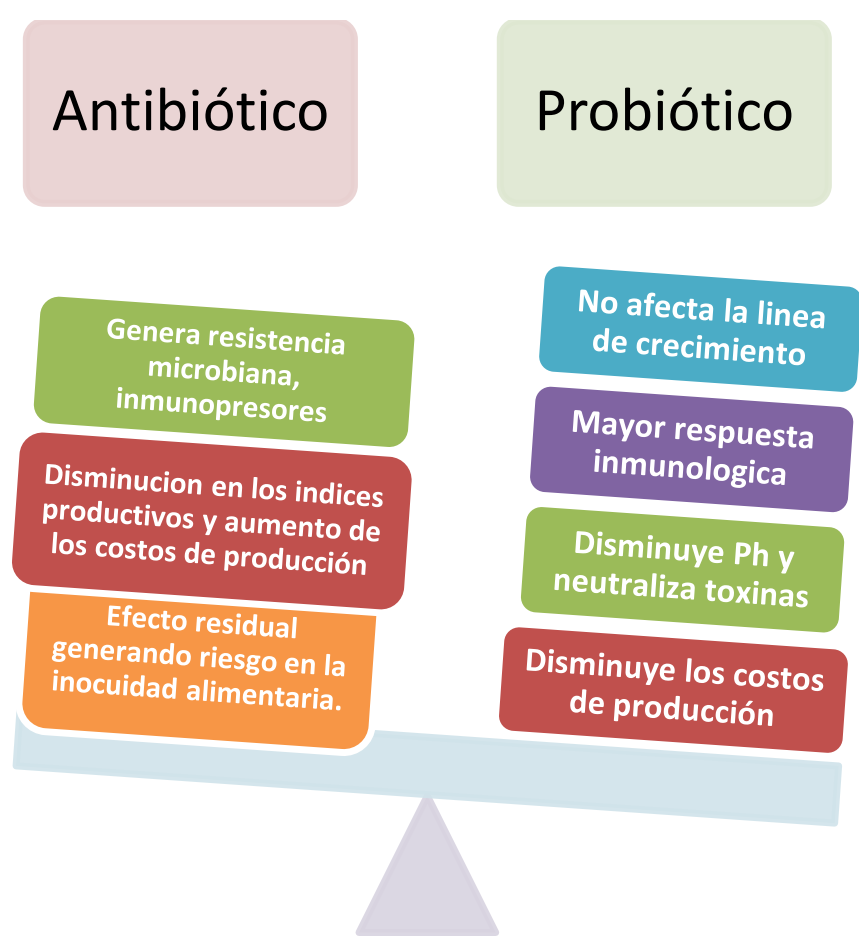

Figura 3. Comparación del uso de Antibiótico y Probióticos (Lara y Burgos, 2012)

\section{ALGUNOS RESULTADOS SOBRE EL USO DE PROBIÓTICOS EN ANIMALES}

Molina, (2008) reporta un aumento de $19.49 \mathrm{~g}$ en ganancia de peso de cuy alimentados con $L$. acidophilus, con respecto al tratamiento testigo, y en pollos de engorde con probiótico $B$. Toyoi un aumento de $64 \mathrm{~g}$ con respecto al tratamiento testigo. En concordancia Torres et al., (2013) demostraron que en esta misma especie de roedor, la adición de Lactobacillus contribuyó con la absorción de nutrientes ya que degrada rápidamente las moléculas grandes para una mayor difusión por la pared intestinal; asimismo demostraron que el índice de conversión alimenticia mejoró hasta un de $3.9 \%$ con el uso de probióticos en la etapa de crecimiento y engorde. 
Lara y Burgos, (2012) evaluaron de forma in vitro el potencial probiótico de cepas nativas aisladas de las heces de pollos asilvestrados en donde demostraron que Saccharomyces sp., Bacillus sp. y Lactobacillus sp. poseen propiedades para ser utilizadas como aditivos microbianos destinados a la alimentación de pollos con el fin de beneficiar su microbiota intestinal e incrementar los índices productivos.

García et al., (2005) observaron una ganancia de peso de 102.3 a 106.8 g y una mejor conversión alimenticia de $1.8 \%$ con respecto al tratamiento control en aves de engorde (Figura 4) adicionando a la dieta S. cerevisiae. Resultados similares obtuvieron Miazzo et al., (2005) en sus investigaciones utilizando S. cerevisiae donde mejoró la ganancia de peso, conversión alimenticia y disminución de la grasa abdominal.

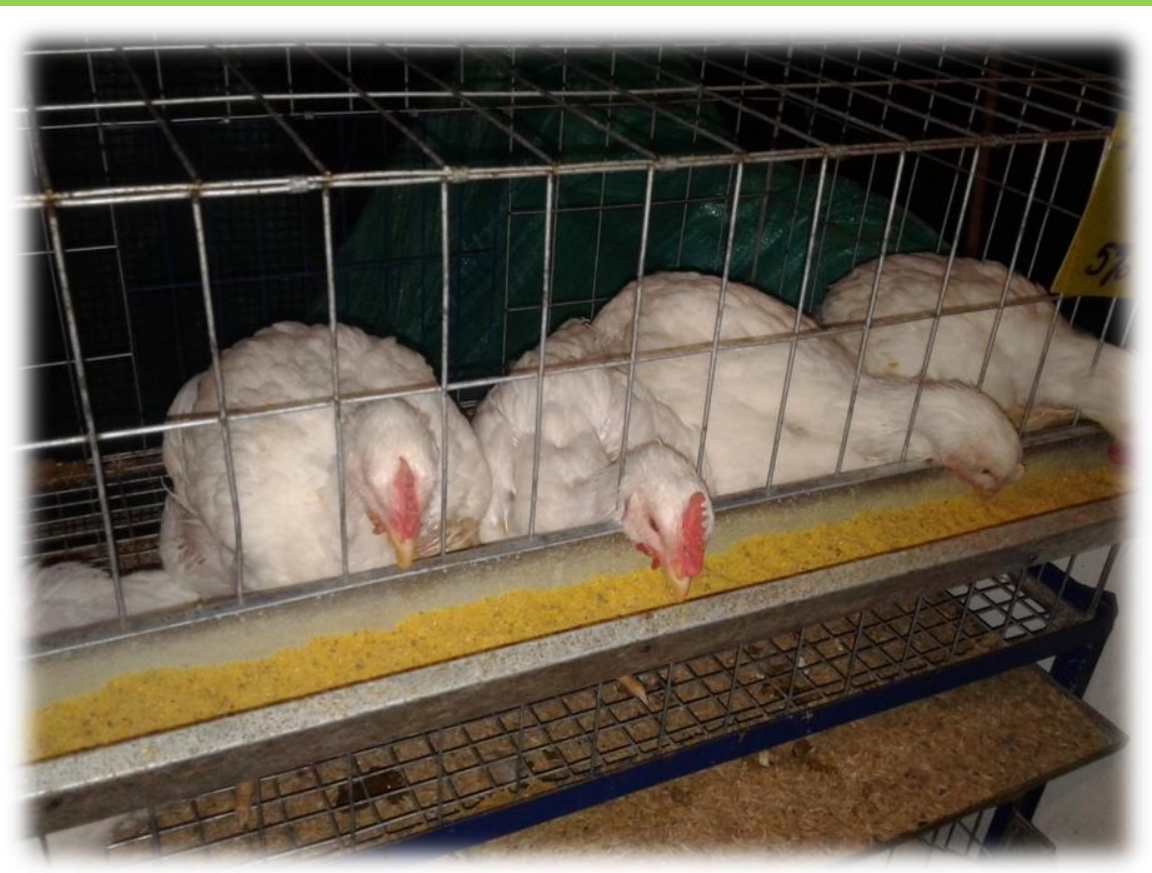

Figura 4. Aves de engorde alimentadas con una dieta balanceada con adición de probióticos. Fuente: Fotografía tomada por Andrea Güecha, (2016).

Osorio et al., (2010) en un estudio comparativo de rendimiento productivo de pollos suplementados con un probiótico comparado con un antibiótico, observaron un $4 \%$ de mayor eficiencia alimenticia en comparación con el grupo control, y $1.6 \%$ en comparación con las aves tratadas con antibióticos, lo cual puede estar dado 
por un incremento en la actividad digestiva, por el uso del probiótico. Estos resultados concuerdan con lo reportado por Cardoso et al., (2011) que realizaron estudios sobre la influencia de un probiótico Vs antibiótico con respecto al rendimiento del pollo (0-21 días de edad), demostrándose un aumento de peso mayor con respecto al tratamiento del antibiótico, aunque la conversión alimenticia no presentó diferencia significativa $(P>0.05)$.

Los probióticos que se han utilizado en conejos y en lechones (Figura 5) mejoran el equilibrio microbiano intestinal, no generan resistencia a patógenos ni residuos indeseables, y contribuyen al control de salmonelosis por su capacidad inmunoestimulante, tal como lo constatan estudios sobre el efecto simbiótico de probióticos, según lo reportado por Chiquieri, (2006) el antibiótico puede ser sustituido por el probiótico $S$. cerevisiae y/o prebiótico manano-oligosacárido, sin perjuicio del desempeño de cerdos en crecimiento y terminación, criados en condiciones tropicales.

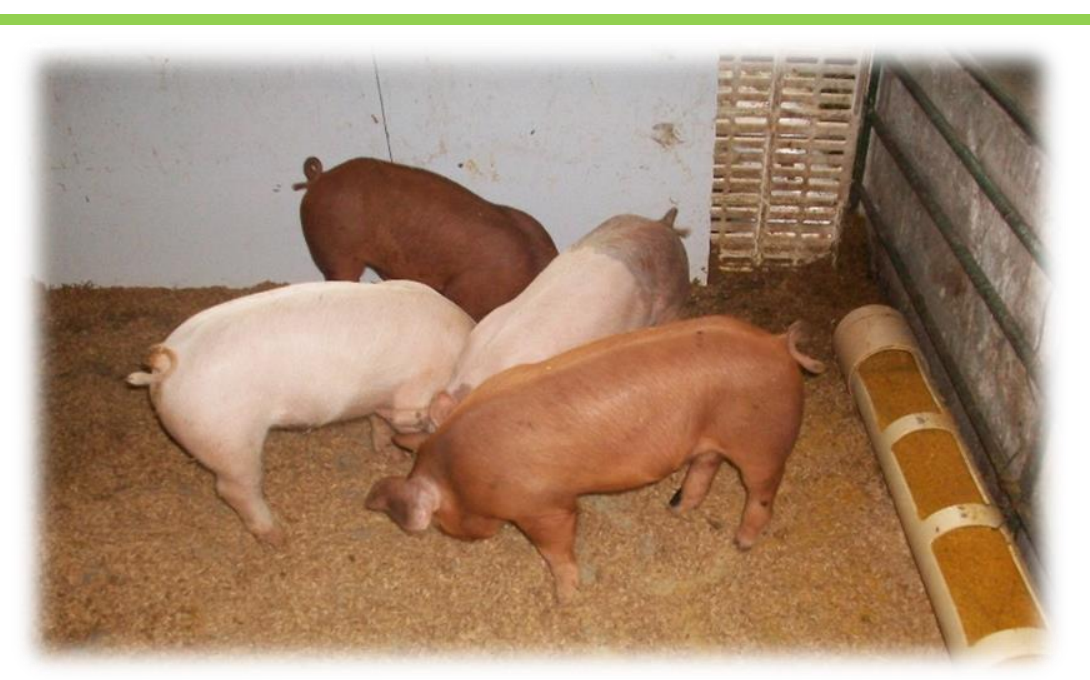

Figura 5. Implementación de dietas balanceadas en lechones. Fuente: Fotografía tomada por Andrea Güechá, (2016).

Ayala, (2008) encontró que los principales rasgos del comportamiento en cerdos con relación al peso vivo $(\mathrm{kg})$, la ganancia de peso $(\mathrm{kg})$ a los 21 días y el peso al destete presentaron diferencias significativas a favor del tratamiento al que se le 
suministró el probiótico (que contenía cepas de L. acidophilus, L. rhamnosus), indicando un efecto positivo en la asimilación de los alimentos.

En terneros se ha observado que la administración de probióticos puede reducir la incidencia de diarreas según lo reportado por Carro, (2014) en corderos se observó que la administración de probióticos aceleró el establecimiento de la población microbiana ruminal, el autor propone que los probióticos pueden favorecer el desarrollo de la microbiota gastrointestinal de animales pre-rumiantes, lo que se puede traducir en una mejora de los índices productivos al reducir la mortalidad y/o morbilidad.

Los mecanismos de acción de los probióticos como las levaduras en ovejas adultas son diferentes, porque estimulan el crecimiento de bacterias celulolíticas desencadenando un mecanismo en el cual se reducen las concentraciones de ácido láctico en el rumen, y de esta forma se disminuye el riesgo de acidosis estabilizando el pH ruminal adecuado para la acción de las bacterias celulolíticas. Como consecuencia de lo anterior se produce un aumento de la degradación de la fibra y la producción de ácidos grasos volátiles, traduciéndose en una mejora de la eficiencia de utilización del alimento (Carro, 2014).

Galina, (2009) determinó que la adición de probióticos de bacterias lácticas (BAL) en la dieta para cabritos en crecimiento puede incrementar la ganancia de peso vivo desde 129 hasta $169 \mathrm{~g}$, así como producir cambios favorables en la digestibilidad, la proteína microbiana y la cinética ruminal.

\section{CONSIDERACIONES FINALES}

En los estudios de investigación realizados hasta ahora sobre la adición de diferentes grupos de probióticos en dietas para animales rumiantes y no rumiantes que se han revisado en este trabajo, los autores coinciden en definir los probióticos como aditivos alimentarios constituidos por microorganismos vivos, que al suministrarse en cantidades adecuadas pueden producir un efecto benéfico en la fisiología digestiva y efectos positivos sobre la salud del hospedero, a partir de la mejora que generan en el equilibrio microbiano del intestino. Por ejemplo, los 
estudios realizados por Molina, (2008) y Torres et al., (2008) en cuyes, coincidieron sobre los beneficios al utilizar probióticos en la dieta, observando un aumento en ganancia de peso, y mejora en la conversión alimenticia en etapas de crecimiento y engorde.

Los resultados sobre el uso de probióticos en aves concuerdan entre lo encontrado por Cardoso et al., (2011), Osorio et al., (2010) y García et al., (2005) donde demuestran que la influencia de un probiótico comparado con el uso de antibiótico, presenta una mayor conversión alimenticia y ganancia en peso con respecto al tratamiento del antibiótico. Al igual que estudios realizados en rumiantes por Galina, (2009) y Carro, (2014), determinaron que la implementación de probióticos en la dieta alimenticia aumenta la ganancia de peso vivo debido a que estos contribuyen al desarrollo de la flora gastrointestinal mejorando los índices productivos tanto en cabras como en corderos.

Por todo lo anterior y dada la importancia de difundir la producción limpia en todas las áreas de producción pecuaria, es conveniente seguir la revisión exhaustiva de la importancia de los probióticos como el futuro para dar solución a algunos de los problemas más recurrentes en sistemas intensivos de producción como son los desbalances causados por bacterias entéricas que provocan disminución en la digestión y absorción de nutrientes y, por lo tanto, retardo en la producción.

\section{CONCLUSIONES}

El uso de probióticos en la industria pecuaria ha ganado amplio terreno en los últimos años, ya que se ha demostrado las características benéficas sobre el hospedero en las diferentes especies animales destinadas a la producción de proteína animal para el consumo humano.

Existe gran potencial en el uso de aditivos probióticos en la dieta para animales y se requieren de estudios que permitan determinar los microrganismos benéficos con posible capacidad probióticas, que permitan llevar a encontrar posibles soluciones con el estado sanitario de los animales y al mismo tiempo generar beneficios en los parámetros productivos. 
El uso de probióticos podría permitir que las empresas cambien a un sistema de producción orgánico o que penetren en el mercado de alimentos buenos para la salud.

\section{REFERENCIAS BIBLIOGRÁFICAS}

1. Ayala L, Bocourt R, Martínez M, Castro M, Hernández L. Respuesta productiva, hematológica y morfométrica de un probiótico comercial en cerdos jóvenes. Revista Cubana de Ciencia Agrícola, 42 (2): 181-184. 2008.

2. Angel M. A. Uso de probióticos en la nutrición de monogástricos como alternativa para mejorar un sistema de producción. Trabajo de Grado Especialista en Nutrición Animal Sostenible. Universidad Nacional Abierta y a Distancia UNAD, Facatativá, Colombia. 97 p. 2013.

3. Bazay G. Uso de los probióticos en la alimentación animal con énfasis en Saccharomyces cerevisiae. p 1-13. 2010. Recuperado 02 Febrero 2016. Disponible En: https://veterinaria.unmsm.edu.pe/files/Articulo bazay Saccharomyces cerevis iae.pdf

4. Blajmana J, Zbruna M, Astesana D, Berisvil A, Romero A, Fusari M, Frizzo L. Probióticos en pollos parrilleros: una estrategia para los modelos productivos intensivos. Revista Argentina de Microbiología, 47 (4): 360-367. 2005.

5. Cardoso L, Cassimira de Silva, C., Duarte Silva, P., Zanardo Donato, D. C., Alburqueque, R., Araùjo, L. Influence of a probiotic on broiler performance. Revista Brasileira de Zootecnia, 40 (12): 2739-2743. 2011.

6. Carro D, Saro C, Mateos I, Díaz A, Ranilla M. Empleo de probióticos en la alimentación de rumiantes. Rev Alimentación Animal. 14: 42-43. 2014. Disponible En: http://oa.upm.es/35230/1/INVE MEM 2014 191170.pdf

7. Figueroa J, Chi E, Cervantes M, Domínguez I. Alimentos Funcionales para cerdos al destete. Revista Veterinaria México, 37 (1): 117-136. 2006.

8. Chiquieri M, Soares T, Souza C, Hurtado V, Ferreira R, Ventura BG. Probiótico y prebiótico en la alimentación de cerdos en crecimiento y terminación. Archivos de Zootecnia, 55 (211); 305-308. 2006.

9. Eicher G. Alternativas na formulacao a dietas vegetarianas para frangos de corte. Tesis de Grado Magister en Zootecnia. Facultad de Agronomia, Universidad Federal do Rio Grando do Sul. 95 p. 2005.

10. Galina $M$, Delgado $M$, Ortíz $M$, Pineda L, Puga D. Cinética ruminal y crecimiento de cabritos suplementados con un probiótico de bacterias ácidolácticas. Pastos y Forrajes, 32 (4): 1-12. 2009.

11. García Y, García Y, Lopez A, Boucourt R. Probióticos: una alternativa para mejorar el comportamiento animal. Revista Cubana de Ciencia Agricolas, 39 (2): 129-140. 2005. 
12. Lara C, Burgos A. Potencial probiótico de cepas nativas para uso como aditivos en la alimentación avícola. Revista Colombiana de Biotecnología, 14 (1): 31-40. 2012.

13. Loddi M, Gonzales E, Sayuri T, Mendes A, de Oliveira R. Uso de probiótico e antibiótico sobre o desempenho, o rendimento e a qualidade de carcaça de frangos de corte. Revista Brasilera de Zootecnia, 29 (4): 1124-1131. 2000.

14. Miazzo R, Peralta M, Picco M. Performance productiva y calidad de canal en broilers que recibieron levadura de cerveza (S. cerevisiae). Revista Electrónica de Veterinaria REDVET, 6 (12): 1-9. 2005. Diponible En: http://www.veterinaria.org/revistas/redvet/n121205/120505.pdf

15. Milian G, Pérez M, Bocourt R. Empleo de probióticos basado en Bacillus sp y de sus endosporas en la producción avícola. Revista Cubana de Ciencias Agrícolas, 42 (2): 117-122. 2008.

16. Molina M. Efecto probiótico de Lactobacillus acidophilus y Bacillus subtilis en cuyes (Cavia porcellus) de engorde. Tesis de Ingeniero Agropecuario. Facultad de Ingeniería de Ciencias Agropecuarias. Escuela Politécnica del Ejército. Sangolquí, Ecuador. 118 p. 2008.

17. Osorio C, Icochea E, Reyna P, Guzmán J, Cazorla F, Carcelén F. Comparación del rendimiento productivo de pollos de carne suplementados con un probiótico versus un antibiótico. Revista de Investigaciones Veterinarias del Peru, 21 (2): 219-222. 2010.

18. Rigobelo E, Maluta R, Ávila F. Desempenho de frangos de corte suplementadas com probiótico. ARS Veterinaria, 27 (2): 111-115. 2011.

19. Samaniego L, Laurencio M, Pérez M, Milián G, Rondón A, Piad R. Actividad probiótica de una mezcla de exclusión competitiva sobre indicadores productivos en pollos de ceba. Ciencia y Tecnología Alimentaria, 5 (5): 360367. 2007.

20. Sarmiento LA. Alimentos funcionales, una nueva alternativa de alimentación. Revista Orinoquia, 10 (1): 16-23. 2006.

21. Torres C, Carcelén F, Ara M, San Martín F, Jiménez R, Quevedo W, Rodríguez J. Efecto de la suplementación de una cepa probiótica sobre los parámetros productivos del cuy (Cavia porcellus). Revista de Investigaciones Veterinarias del Perú, 24 (4): 433-440. 2013. 